\title{
A lattice-Boltzmann approach for the modeling of microscopic three-phase flow mechanisms
}

\author{
van Kats, Florian M., Egberts, Paul J.P. and Floris, Frans J.T \\ TNO Institute of Applied Geoscience, Delft, The Netherlands
}

Paper presented at the 5th European Conference on the Mathematics of Oil Recovery, Leoben, Austria, 3-6 Sept. 1996

\begin{abstract}
Utilizing a recently developed numerical technique, known as the lattice-Boltzmann method, we study immiscible three-phase flow at the pore scale. An important phenomenon at this scale is the spreading of oil onto the gas-water interface. In case of a spreading oil droplet, it is shown that the simulated flow dynamics agree with an analytical model.

We also simulate steady-state three-phase flow in a small segment of a 2D waterwet porous medium under both spreading and non-spreading conditions. The difference between the two conditions with respect to the phase mobilities in the generalized law of Darcy (which also accounts for viscous coupling) is investigated.
\end{abstract}

\section{INTRODUCTION}

Three-phase flow in porous media is an important process in enhanced oil recovery and in pollutant clean-up. Gas injection is one of the most widely applied methods in this context. For the associated immiscible three-phase flow, several macroscopic models have been proposed. These models are either based on two-phase flow data or they rely on fits to experimental three-phase data [Stone, 1973; Baker, 1988]. It has been shown that such models are not reliable in the prediction of the large scale behaviour. To obtain reliable models, many authors [Chatzis et al., 1988; Baker, 1988; Oak, 1990] have stressed to take into account pore level physics. It has been recognized that the spreading of oil onto the gaswater interface is an important displacement mechanism in waterwet porous media [Øren and Pinczewski, '1995; Kalaydjian et al., 1993]. We will focus in particular on this three-phase phenomenon.

The so-called spreading coefficient $S$ with respect to the oil phase is a parameter which determines whether oil is able to form spreading films between water and gas. This coefficient is given by

$$
S=\gamma_{w g}-\gamma_{w o}-\gamma_{o g}
$$

where $\gamma_{w \mathrm{~g}}, \gamma_{\mathrm{wo}_{\mathrm{o}}}$ and $\gamma_{\mathrm{og}}$ are the interfacial tensions for respectively the water-gas, water-oil and oil-gas interface. In this paper we will assume, for convenience, that the inequalities $\gamma_{w o}<\gamma_{w_{g}}+\gamma_{o g}$ (gas cannot spread between water and oil) and $\gamma_{o g}<\gamma_{\text {wg }}+\gamma_{w o}$ (water cannot spread between gas and oil) hold. For negative values of $S$, spreading of oil does not occur. In that case non-zero contact angles will be observed as shown schematically in figure $1(\theta>0)$. For non-negative values of $S$, spreading of oil $(\theta=0)$ occurs whenever three-phase contacts are created. However, the spreading process may stop after thermodynamic equilibrium has been reached. This is due to the fact that $S$ will either be zero or negative in thermodynamic equilibrium Rowlinson and Widom, 1982].

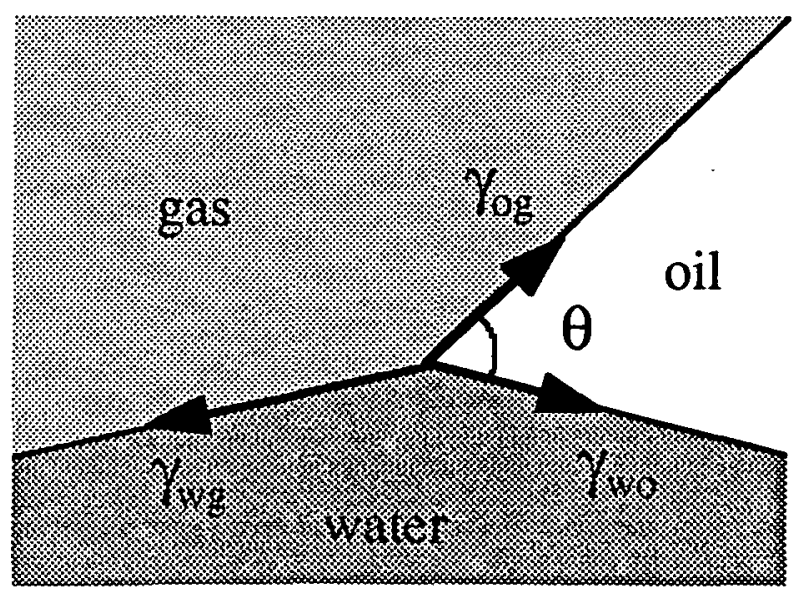

Figure 1: Balance of interfacial tensions where three phases are in contact $(S<0)$. 
Spreading oil films may become connected over large distances in waterwet porous media, such that the oil tends to flow as a continuous phase. For that reason it is expected that spreading of oil improves the oil recovery, which has been confirmed by means of simulations [Øren and Pinczewski, 1995] and experiments [Kalaydjian et al., 1993].

Of crucial importance for multi-phase flow models is knowledge of how the different phases are distributed in a porous medium (e.g. spreading or non-spreading oil). In order to avoid assumptions about how three immiscible phases are distributed on the pore level, it is required to use models which capture immiscible three-phase flow on this level. For that purpose, we utilize a recently developed numerical method, known as the latticeBoltzmann method. This method originates from latticegas techniques for simulating single and multi-phase flow [Frisch et al., 1987; Gunstensen and Rothman, 1991]. The lattice-gas method models a fluid as a collection of discrete particles of unit mass and momentum moving from site to site along the edges of a regular lattice. When particles reach a site, they undergo a collision. When the collisions are chosen to conserve mass and momentum, it can be shown that the model obeys the Navier-Stokes equations of incompressible hydrodynamics in an asymptotic limit [Frisch et al., 1987]. The numerical results, however, are noisy due to the discrete character of the lattice-gas model. To obtain smooth hydrodynamics, it is necessary to average over long time intervals when the flow has reached a steady-state. This makes the lattice-gas method unsuitable to model non-steady state flow.

The lattice-Boltzmann method resolves this problem by modeling a fluid as an average of particles rather than the discrete particles themselves. It solves the full incompressible Navier-Stokes equations [Chen et al., 1992; Grunau, 1993],

$$
\begin{gathered}
\nabla \cdot \underline{u}=0 \\
\frac{\partial \underline{u}}{\partial t}+\underline{u} \cdot \nabla \underline{u}=-\nabla p+v \nabla^{2} \underline{u}
\end{gathered}
$$

where $\underline{u}$ is the average particle velocity, $p$ is the isotropic pressure, and $v$ is the kinematic viscosity. The method is capable of implicitly tracking fluid-fluid interfaces; at an interface, particles of two phases (usually painted red and blue) exist and are redirected to their own phase in order to simulate interfacial tension [Gunstensen et al., 1991]. Another important feature of the lattice-Boltzmann method is that fluid-solid interfaces are easily handled with respect to shape (geometry), no-slip and wettability.

We use a three-phase (2D) lattice-Boltzmann model in which the technique to simulate fluid-fluid interfaces is taken from the model of Gunstensen et al. [1991]. With this three-phase model, fundamental laws have been confirmed. In particular, simulation results involving the Laplace law of capillary pressure, three-phase contact angles (such as in figure 1) and spreading behaviour closely follow the corresponding theory.
In this paper we will briefly describe the three-phase lattice-Boltzmann model. The spreading of oil onto a gaswater interface is simulated. The result of this simulation is compared with an analytical model and experiments. Further, we will investigate the impact of oil spreading on the phase mobilities in the generalized law of Darcy which accounts for viscous coupling.

\section{NUMERICAL MODEL}

\section{Single-Phase Model}

The lattice-Boltzmann model, we will describe, represents a flow domain by a hexagonal lattice (see figure 2a). At each lattice site (node) $\underline{x}$ six unit velocity vectors $\mathrm{e}_{\mathrm{i}}$ are defined by:

$$
\underline{e}_{i}=\left[\begin{array}{c}
\cos (\pi(i-1) / 3) \\
\sin (\pi(i-1) / 3)
\end{array}\right], \quad i=1, \ldots, 6
$$

The construction is such that $\underline{x}+\underline{e}_{i}$ are neighbour sites of $\underline{x}$ (see figure $2 b$ ).

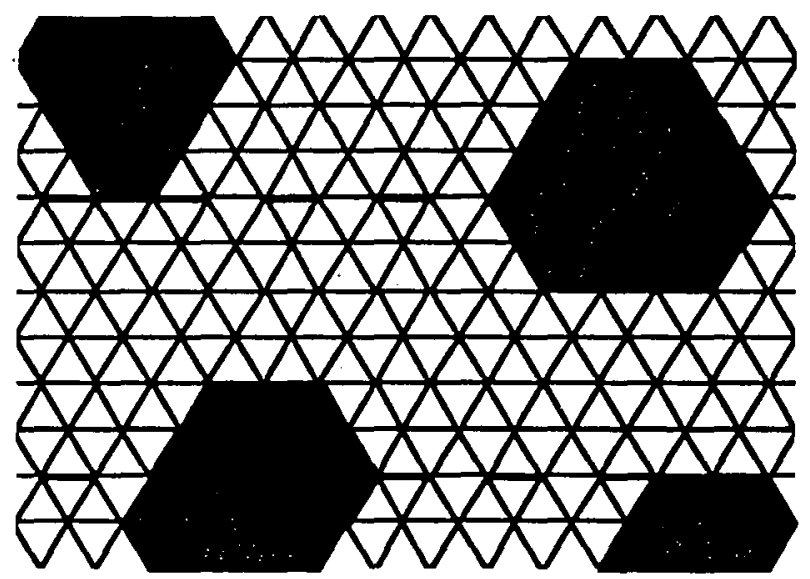

Figure 2a: Segment of a hexagonal lattice on which grains (black regions) are mimicked.

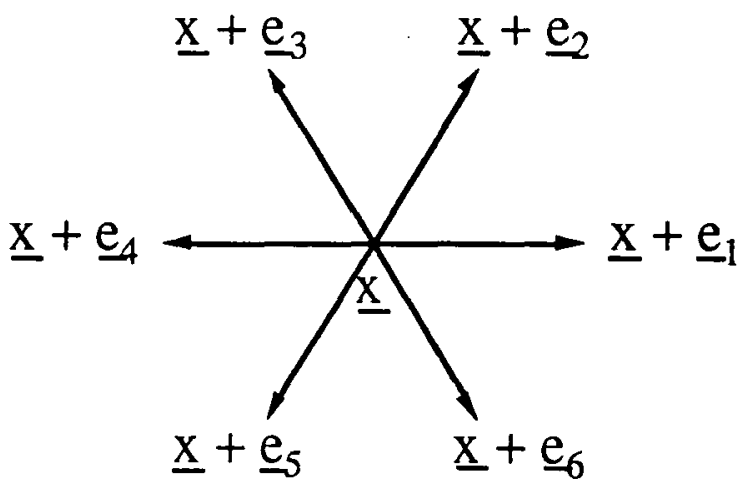

Figure $2 b$ : Site $\underline{x}$ and its six neighbour sites.

We recall that in the lattice-gas method, discrete particles move from site to site along the edges of the lattice. 
The lattice-Boltzmann method replaces the discrete particles by particle distributions. These distributions, present at site $\underline{x}$ and discrete time step $t$, are denoted by $\mathrm{N}_{\mathrm{i}}(\mathrm{x}, \mathrm{t})$ and $\mathrm{can}$ be understood as the fraction of fluid particles which travel from site $\underline{x}$ with velocity $\mathrm{e}_{i}$. The model we consider also accounts for a fraction of rest particles $N_{0}(x, t)$ with velocity $e_{0}=0$ (from now on the index $i$ will take the values $0,1, \ldots, 6)$. The local mass density $\rho$ and momentum $\rho \underline{u}$ are defined by:

$$
\rho \equiv \sum_{i} N_{i} \text { and } \rho \underline{u} \equiv \sum_{i} \underline{e}_{i} N_{i}
$$

The evolution of the system is described by the Boltzmann equation

$$
N_{i}\left(\underline{x}+\underline{e}_{i}, t+1\right)=N_{i}(\underline{x}, t)+\Delta_{i}(\underline{x}, t)
$$

in which $\Delta_{\mathrm{i}}$ is the collision operator. Introducing a free parameter $\tau$, the system can be relaxed to equilibrium on a single time scale by assigning

$$
\Delta_{i}=-\frac{1}{\tau}\left\{N_{i}-N_{i}^{e q}\right\}
$$

where $N_{i}^{e q}=N_{i}^{e q}(\rho(\underline{x}, t), \underline{u}(\underline{x}, t))$ are the equilibrium distributions which arise from earlier developments of latice-gas techniques, as shown by Frisch et al. [1987]. The collision operator satisfies conservation of mass and momentum:

$$
\begin{aligned}
& \sum_{i} \Delta_{i}=0, \text { so } \quad \sum_{i} N_{i}^{e q}=\rho \\
& \sum_{i} \underline{e}_{i} \Delta_{i}=\underline{0} \text {, so } \sum_{i}^{i} \underline{e}_{i} N_{i}^{e q}=\rho \underline{u}
\end{aligned}
$$

The lattice-gas model of Frisch et al. [1987] and related models possess some rather unphysical effects. These are a non-Galilean invariance and a velocity-dependent equation of state. Here, the freedom exists to construct the equilibrium distributions such that these effects are eliminated. A detailed discussion about this is given by Chen et al. [1992].

A continuous approximation of the Boltzmann equation (5) can be made by performing a Taylor series expansion, after which, in an asymptotic limit, the incompressible Navier-Stokes equations (2) are recovered [Chen et al., 1992; Grunau, 1993]. This analysis shows that the relaxation parameter $\tau$ determines the kinematic fluid viscosity as follows:

$$
v=\frac{(2 \tau-1)}{8}
$$

\section{Multi-Phase Model}

For two- and three-phase systems of immiscible fluids, it is required to model interfacial tension dynamics. The different fluid phases will be denoted by $k$, where $k=$ $r$ (red), $k=b$ (blue) or $k=g$ (green). For each separate phase, quantities like mass densities and equilibrium distributions are determined similarly as in the singlephase model. In single-phase regions, the system evolves in the same way as before:

$$
N_{i}^{(k)}\left(\underline{x}+\underline{e}_{i}, t+1\right)=N_{i}^{(k)}(\underline{x}, t)+\Delta_{i}^{(k)}(\underline{x}, t)
$$

where

$$
\Delta_{i}^{(k)}=-\frac{1}{\tau_{k}}\left\{N_{i}^{(k)}-N_{i}^{(k) e q}\right\}
$$

is the collision operator as in the single-phase model. Hence, the incompressible Navier-Stokes equations are satisfied within each phase.

At sites where more than one phase exists, a procedure is applied to model fluid-fluid interfaces and three-phase intersections. This procedure consists of two steps (see also Gunstensen et al. [1991], and Grunau [1993] for twophase flow), being a perturbation step and a recolouring step. In the perturbation step, an anisotropic perturbation is added to the particle distributions. This perturbation tunes the magnitude of interfacial tensions. In the recolouring step, the particle distributions are redinected such that the phases remain separated.

At interfaces and three-phase intersections, the updating of the particle distributions (perturbation step) is described by

$$
\begin{aligned}
\tilde{N}_{i}^{(k)}(\underline{x}, t) & =N_{i}^{(k)}(\underline{x}, t)+ \\
& +\Delta_{i}^{(k)}(\underline{x}, t)+\Omega_{i}^{(k)}(\underline{x}, t)
\end{aligned}
$$

where

$$
\Omega_{i}^{(k)}=A_{k} \frac{\left|\underline{e}_{i} \| \underline{F}^{(k)}\right|}{2}\left\{\frac{\left(\underline{e}_{i} \cdot \underline{F}^{(k)}\right)^{2}}{\left|\underline{F}^{(k)}\right|^{2}}-\frac{1}{2}\right\}
$$

is the perturbation operator which conserves mass and momentum. The vectors $E^{(k)}$ in (12) are called colour vectors. They contain information about the orientations of the fluid-fluid interfaces by taking into account the mass densities at the neighbour sites. For that purpose, the following vectors $\mathrm{f}^{(\mathrm{k})}$ are defined:

$$
\underline{f}^{(k)}=s_{k} \sum_{i} \underline{e}_{i} \rho_{k}\left(\underline{x}+\underline{e}_{i}, t\right)
$$

in which $s_{k}$ are free parameters. The colour vectors ane linear combinations of the vectors $\mathrm{f}^{(k)}$ :

$$
\begin{aligned}
& E^{(r)}=\underline{f}^{(r)}-\underline{f}^{(b)}-\underline{f}^{(g)} \\
& F^{(b)}=f^{(b)}-\underline{f}^{(r)}-\underline{f}^{(g)} \\
& \bar{F}^{(g)}=\underline{f}^{(8)}-\underline{f}^{(r)}-\underline{f}^{(b)}
\end{aligned}
$$

At a red-blue interface (where $\underline{f}^{(\overline{)}}=Q$ ), for example, the rod colour vector $\underline{F}^{(r)}$ will be perpendicular to the interface and pointing into the red phase. The blue colour vector (of equal size) will point into the opposite direction, also perpendicular to the interface. In that case, the perturbation operator is applied for both the red phase and the blue phase; basically, it redistributes momentum by depleting momentum along lattice links parallel to the interface and adding momentum to lattice links perpendicular to the interface. This redistribution reflects the repulsive force between the two phases, which simulates interfacial tension.

At sites where the three phases coexist, the perturbations occur in such a way that there is a continuous transition to the two-phase perturbations. The constants $A_{k}$ are 
arbitrary. However, they must be chosen sufficiently small in order to obtain perturbations which are small compared to the particle distributions. In this model, we will assume $A_{7}=A_{b}=A_{g}=A$.

After the collisions and perturbations have taken place, the particle distributions need to be redirected (recolouring step) in order to remain the immiscibility of the fluid phases. More precisely, the recolouring step consists of maximizing

$$
\sum_{i} \sum_{k} \hat{N}_{i}^{(k)} \underline{e}_{i} \cdot \underline{F}^{(k)}
$$

subject to the following constraints:

$$
\begin{aligned}
\sum_{i} \hat{N}_{i}^{(k)} & =\sum_{i} \tilde{N}_{i}^{(k)} \\
\sum_{k} \hat{N}_{i}^{(k)} & =\sum_{k}^{i} \tilde{N}_{i}^{(k)}
\end{aligned}
$$

These constraints represent respectively conservation of mass and momentum; the total mass of each individual phase as well as the total mass at each lattice link are conserved.

The evolution equation is completed by the straightforward propagation:

$$
N_{i}^{(k)}\left(\underline{x}+\underline{e}_{i}, t+1\right)=\hat{N}_{i}^{(k)}(\underline{x}, t)
$$

The values of the interfacial tensions can be approximated theoretically by analyzing the perturbation step. The derivation has been given by Gunstensen et al. [1991] who utilized the mechanical definition of the interfacial tension. Here, a slightly altered analysis results into

$$
\gamma_{k l} \approx \frac{9}{4} A\left\{\rho_{k} s_{k}+\rho_{l} s_{l}\right\}\left\{\frac{\rho_{k} \tau_{k}+\rho_{l} \tau_{l}}{\rho_{k}+\rho_{l}}\right\}
$$

where $\gamma_{k b}$ is the interfacial tension between any two of the three pairs of fluids.

In expression (18), $\rho_{\mathbf{k}}$ is the mass density in the interior of phase k. A fundamental problem arises if density differences are allowed, for then the velocity will no longer be continuous across a fluid-fluid interface [Ginzbourg, 1994]. Therefore, we will assume $\rho_{\mathrm{r}}=\rho_{\mathrm{b}}=\rho_{\mathrm{g}}$ $=\rho$ in this model.

The parameters $S_{k}$ in (18) are important in the case of three-phase systems and can be understood as weighting factors which allow the interfacial tension between any two of the three pairs of fluids to be varied relative to the other interfacial tensions. In this way, the interfacial tensions are assigned values independent of the values of the different viscosities which depend only on the $\tau_{k}$. This property is not shared by all lattice-Boltzmann models [Ginzbourg, 1994]. The idea of using weighting factors is also known in the context of three-phase lattice-gas modeling [Gunstensen and Rothman, 1991].

\section{SIMULATION OF SPREADING DYNAMICS}

The understanding of spreading dynamics in the context of three-phase fluid flow has been greatly improved in the last decade. Experimental studies [Fraaije and Cazabat, 1989; Gaver III and Grotberg, 1992] as well as mathematical models [Borgas and Grotberg, 1988; Koch and Koch, 1995] contributed to the current level of knowledge.

Here, we aim to simulate a 2D capillary driven three-phase flow in which spreading occurs. For that purpose, we consider an oil droplet which is brought in contact with a flat gas-water interface. In the vertical direction, the flow domain is bounded by solid walls. In the horizontal direction, a periodic boundary condition is used. The spreading coefficient $S$ is fixed at a value of zero. External forces such as gravity are not imposed. An example of a simulation is shown in figure 3 .

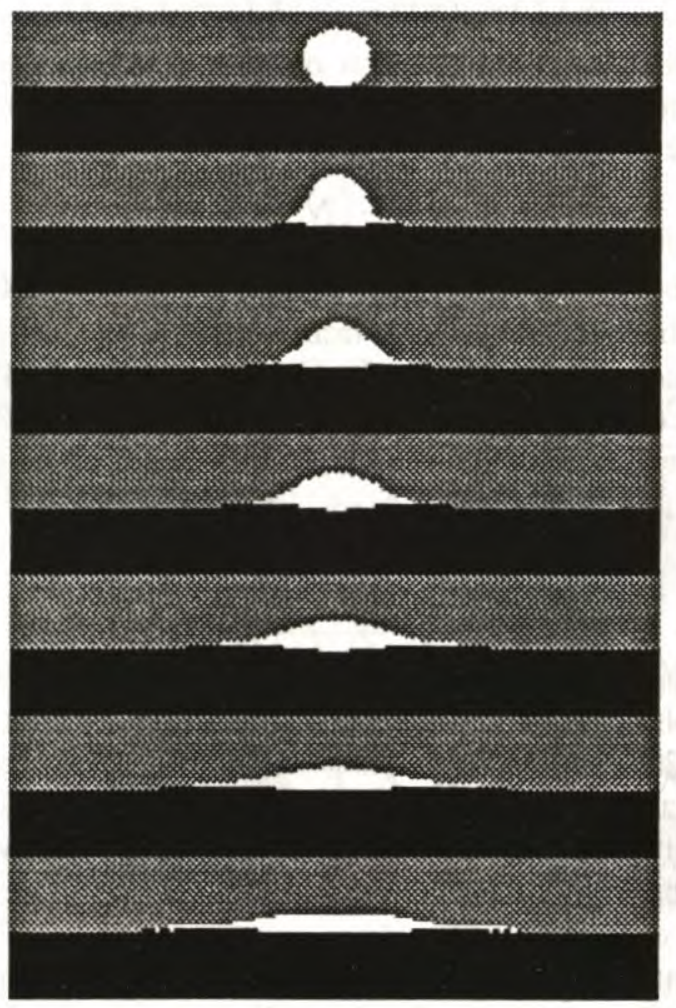

Figure 3: Spreading oil droplet $(S=0)$ at time steps 0 , $1500,3000,5000,10000,15000$ and 25000.

Two time intervals, the first and the second spreading regime, between which a sharp transition exists have been observed. In both regimes, the radius $R(t)$ of the droplet follows a power law:

$$
R(t) \sim t^{\lambda}
$$

\section{The First Spreading Regime}

In the first spreading regime, the radius $\mathrm{R}(\mathrm{t})$ of the droplet is defined as the distance from the center to the end point of the droplet. From the simulations it has been found that the exponent $\lambda$ equals a value of $0.42 \pm 0.02$ in this regime (see figure 4 for an example).

Fluid parameters turn out to affect the velocity of spreading, not the exponent; larger viscosities and/or smaller interfacial tensions (keeping $\mathrm{S}=0$ fixed) slow 
down the velocity of spreading, thereby leaving $\lambda$ unaltered.

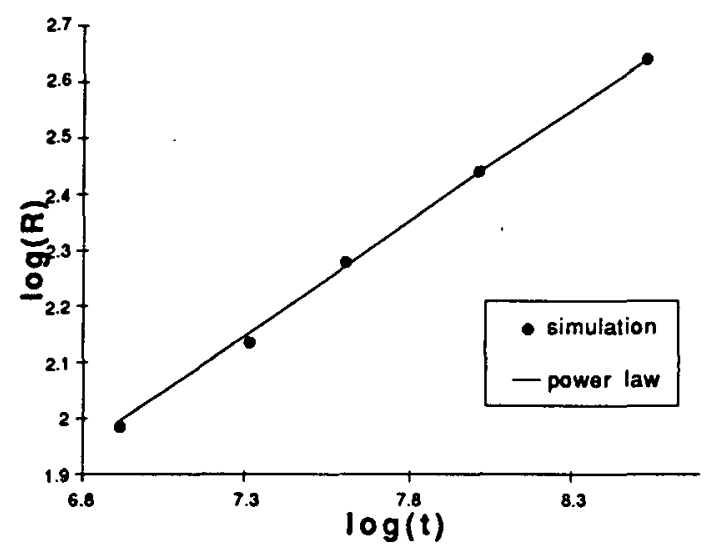

Figure 4: Spreading rate of an oil droplet. The line with slope $\lambda=0.42$ optimally fits the simulation.

The results agree well with results of IFP where an exponent of 0.41 has been obtained from laboratory experiments [Kalaydjian, private communication]. Note that in the simulations, in contrast with the experiments, both gravity as well as contributions from the third dimension are not present.

The capillary force which is the driving force induces two other dominating forces, an inertia force and a viscous force. This has been verified by estimating the ratio of these forces $(\approx O(1))$ numerically, expressed as a dimensionless number (the Reynolds number). Similar estimates indicate that inertia can be neglected in a later stadium of the spreading process.

\section{The Second Spreading Regime}

During the period in the first spreading regime, the oil droplet behaves as a bulk fluid. This is no longer true after a certain period of time because a thin film will develop ahead of the central drop. It has been suggested by Fraaije and Cazabat [1989] to consider an effective radius as a (horizontal) measure for the central part of the drop. They mentioned that such an effective radius may be defined as the distance from the center to the inflection point, i.e. where the curvature changes sign (see figure 5). Here, this definition for $R(t)$ will be used in the second spreading regime.

Simulation results will be compared with results obtained from an analytical model for the spreading rate. The model we will present below is mainly based on the work of Joanny [1987].

The configuration of the fluid phases (at a certain time step $t$ ) is depicted in figure 5. The flow is symmetric in the line $\mathrm{x}=0$. In the vertical direction, the fluid domain is bounded by a horizontal wall at $y= \pm H$. For the sake of simplicity, the interfacial tensions are chosen such that $\gamma_{w_{0}}=\gamma_{o g}=\gamma_{w g} / 2=\gamma$. For convenience, we also assume $\mu_{w}=\mu_{8}=\mu$, where $\mu_{k}$ represents the dynamic viscosity of phase $k$. With these assumptions, the flow can be regarded symmetric also in the line $y=0$ after some period of time. Hence, only the region $0 \leq x \leq R(t), 0 \leq y \leq H$ needs to be considered. The total occupied oil volume (area in 2D) is constant and denoted by $\Omega$.

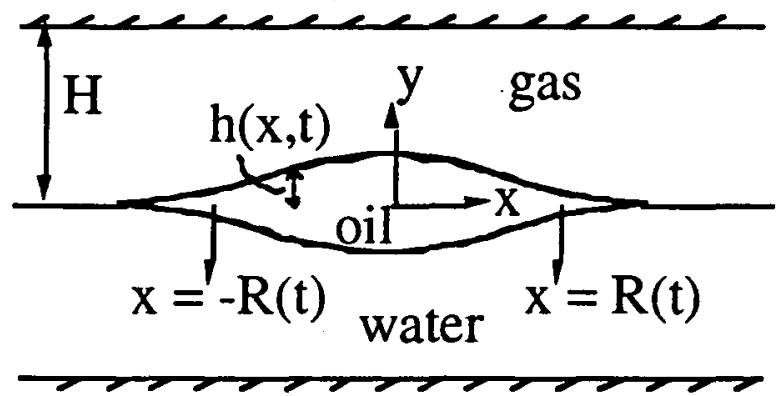

Figure 5: Spreading oil droplet in the second spreading regime.

Since numerical estimates indicated that inertia can be neglected in a later stadium of the spreading process, we assume that the flow is controlled by capillary and viscous forces only. Consequently, the Navier-Stokes equations (2) reduce to the Stoke's equations. For the oil phase this means

$$
\begin{gathered}
\nabla \cdot \underline{u}_{o}=0 \\
\nabla p_{o}=\mu_{o} \nabla^{2} \underline{u}
\end{gathered}
$$

where $p_{o}$ represents the oil pressure and $\underline{u}_{o}$ the oil velocity with horizontal component $u_{0}$.

Another assumption in this model (a lubrication approximation) is that the slope of the curved interface is sufficiently small, i.e. $\left|h_{x}\right| \& 1\left(h_{x}\right.$ denotes the derivative of $h$ with respect to $x$ ). This will be the case when the relative height $h(x=0, t) / R(t)$ becomes small enough. Then, the magnitude of the interface curvature can be approximated by $h_{x x}$.

Further, it is assumed that the velocity profile is linear in the gas and water phase, thereby neglecting the influence of the vertical flow due to the decrease of the height. Taking into account the above assumptions, it follows:

$$
\begin{gathered}
\frac{\partial p_{o}(x, t)}{\partial x}=-\gamma \frac{\partial^{3} h(x, t)}{\partial x^{3}} \text { and } \\
\left|\frac{\partial^{2} u_{o}(x, y, t)}{\partial x^{2}}\right| \ll\left|\frac{\partial^{2} u_{o}(x, y, t)}{\partial y^{2}}\right|
\end{gathered}
$$

Using (21), the horizontal component of the conservation of momentum, described by the Stoke's equations (20), is expressed as

$$
\mu_{o} \frac{\partial^{2} u_{o}(x, y, t)}{\partial y^{2}}+\gamma \frac{\partial^{3} h(x, t)}{\partial x^{3}}=0
$$

which, after integrating with respect to $y$ over the interval $[0, h(x, t)]$, results into 


$$
\mu_{o} \frac{\partial u_{o}(x, y=h(x, t), t)}{\partial y}=-y h(x, t) \frac{\partial^{3} h(x, t)}{\partial x^{3}}
$$

This equation reflects the local balance between the capillary and the viscous force.

The aim is to find an expression for $R(t)$. For that purpose, we introduce the mean velocity $U(x, t)$ defined by

$$
U(x, t)=\frac{1}{h(x, t)} \int_{0}^{h(x, t)} u_{o}(x, s, t) d s
$$

The velocity profile in the gas and water phase has been assumed linear. If it is also assumed that the channel width is much larger than the thickness of the spreading droplet, i.e. $h(0, t) \ll H$, the viscous drag can be calculated by

$$
\mu_{o} \frac{\partial \imath_{o}(x, y=h(x, t) t)}{\partial y}=-\mu \frac{U(x, t)}{H}
$$

which results from the continuity of tangential stress. Substituting (25) into equation (23) gives the integrated horizontal momentum balance:

$$
\mu \frac{U(x, t)}{H}=\operatorname{hr}(x, t) \frac{\partial^{3} h(x, t)}{\partial x^{3}}
$$

We now look for self-similar solutions of equation (26) for $\mathrm{U}(\mathrm{x}, \mathrm{t})$ and $\mathrm{h}(\mathrm{x}, \mathrm{l})$ :

$$
\begin{aligned}
& U(x, t)=v(t) w(\eta) \\
& h(x, t)=f(t) g(\eta)
\end{aligned} \quad 0 \leq \eta \equiv \frac{x}{R(t)} \leq 1
$$

where $v, w, f$ and $g$ are bounded and sufficiently smooth functions. By definition we have

$$
U(x=R(t), t)=\frac{d R}{d t}, \text { hence } v(t)=\frac{d R}{d t}
$$

after having set $w(1)$ equal to 1 .

The total amount of oil is conserved. Here, the oil volume beyond the inflection point is assumed to be negligible compared to the total oil volume (simulation results have shown that this is not unreasonable). Hence, the conservation of mass is written as

$$
\int_{0}^{R(t)} h(s, t) d s=\frac{\Omega}{4}
$$

which results into

$$
f(t)=\frac{1}{R(t)} \text { and } \int_{0}^{1} g(s) d s=\frac{\Omega}{4}
$$

The local mass conservation is expressed by

$$
\frac{\partial h}{\partial t}+\frac{\partial(U h)}{\partial x}=0
$$

Substitution of (27) and the functions $v$ and f from (28) and (30) into the partial differential equation (31), leads to the ordinary differential equation:

$$
\frac{d(w g)}{d \eta}=\frac{d(\eta g)}{d \eta}
$$

Utilizing that $g(\eta)$ is bounded, together with $w(1)=1$, it follows that $w(\eta)=\eta$.
Recollecting the above yields

$$
\begin{gathered}
U(x, t)=\eta \frac{d R}{d t}, \quad 0 \leq \eta \equiv \frac{x}{R(t)} \leq 1 \\
h(x, t)=\frac{g(\eta)}{R(t)} \text { with } \quad \int_{0}^{1} g(s) d s=\frac{\Omega}{4}
\end{gathered}
$$

Using (33), equation (26) can be written as

$$
R^{5}(t) \frac{d R}{d t} \eta=\frac{\gamma H}{\mu} g(\eta) \frac{d^{3} g}{d \eta^{3}}
$$

Solving equation (34) for $R(t)$, after the equation has been integrated with respect to $\eta$ from 0 to 1 , finally results into

$$
\left.R(t)=\varnothing \frac{\gamma H \Omega^{2} t}{\mu}\right]^{\frac{1}{6}}
$$

for sufficiently large $t$. The prefactor $c$ is a positive dimensionless constant, given by

$$
c=\left[\frac{12}{\Omega^{2}} \int_{0}^{1} g(s) \frac{d^{3} g}{d s^{3}} d s\right]^{\frac{1}{6}}
$$

The simulations reveal that, in the second spreading regime, the effective radius indeed follows a power law with an exponent close to $1 / 6$. Taking into account errors in the measurements, we estimate a maximal error of \pm 0.04 for the exponent. The interfacial tension $\gamma$, the viscosity $\mu$ (of the gas and water phase) and the oil viscosity $\mu_{\mathrm{o}}$ have been assigned different values. It has been found that the simulated spreading rates follow the predictions, i.e. equation (35), fixing the prefactor $c$ at 0.87 . This value for $c$ optimally fits one of the simulations. So, the remaining simulations are compared to (35) without fitting any parameter. Note that, according to (35), the oil viscosity does not affect the spreading process. This has been confirmed by the numerical results (see figure 8). Other results are shown in figures 6 and 7. One should observe the transition from the first to the second spreading regime.

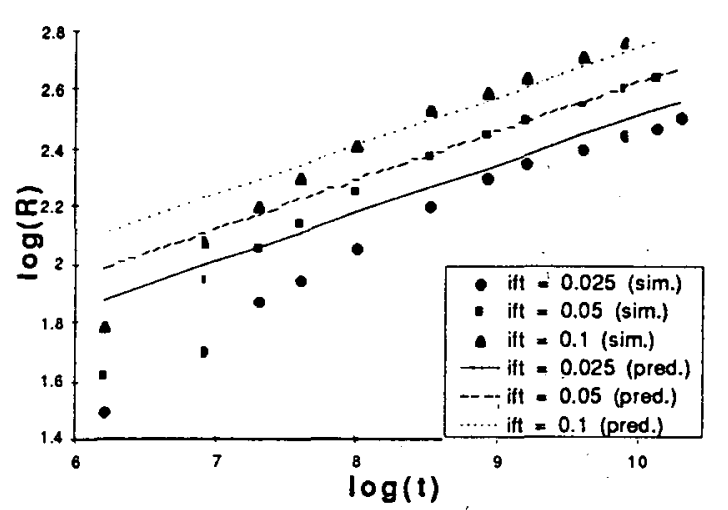

Figure 6: Predicted spreading rates (lines) and simulations (marks) for $\gamma$ (ift) $=0.025,0.05$ and 0.1 (in latticeBoltzmann units), where all other parameters are kept fixed. 


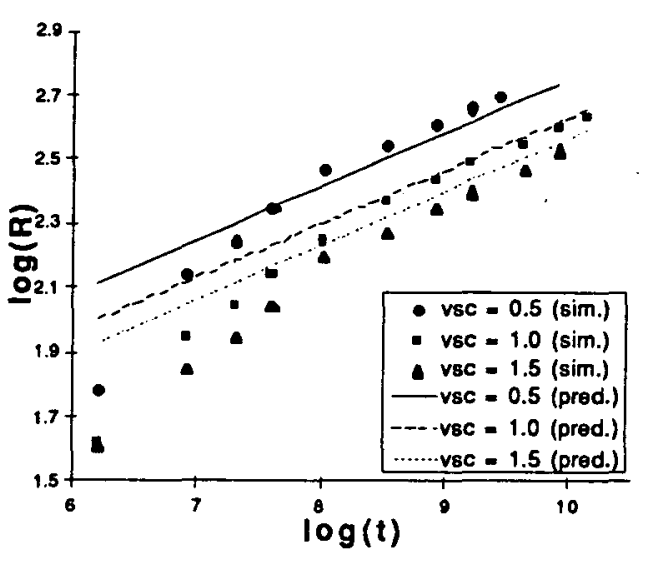

Figure 7: Predicted spreading rates (lines) and simulations (marks) for $\mu$ (vsc) $=0.5,1.0$ and 1.5 (in latticeBoltzmann units), where all other parameters are kept fixed.

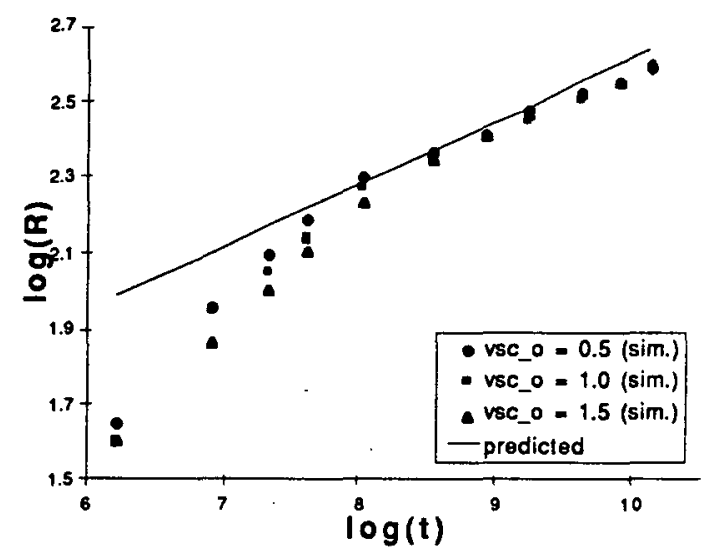

Figure 8: Predicted spreading rate (line) and simulations (marks) for $\mu_{0}$ (vsc_o) $=0.5,1.0$ and 1.5 (in latticeBoltzmann units), where all other parameters are kept fixed.

Fraaije and Cazabat [1989] experimentally observed a power law regarding an effective radius as being the distance from the center to the first interference fringe. They discussed the difficulty to measure other defined effective radii. They also found that the central drop (bulk fluid) and the precursor film (ahead of the central drop) are not hydrodynamically coupled. This justifies the assumption that the effective radius only depends on the flow in the bulk fluids. For a certain time interval in which the droplet has become sufficiently flat (thus neglecting gravitational effects), an exponent of $1 / 6$ fits their measurements (in 3D) closely where theory [Joanny, 1987] predicts an exponent of $1 / 7$ in 3D. Since we found an exponent of $1 / 6$ (theoretically and numerically), this seems to indicate that the exponent in a 2D flow does not differ much from the exponent in a 3D flow.
SIMULATION OE THREE-PHASE FLOW THROUGH A POROUS MEDIUM

Immiscible multi-phase flow at the pore scale is described by the Navier-Stokes equations (2), together with the interfacial tension dynamics. At a macroscopic (volume-averaged) scale, it is generally assumed that the average velocity of a fluid, flowing through a porous medium, depends linearly (in an appropriate flow regime) on both the pressure gradient and a body force (gravity). This linear relationship is known as Darcy's law.

In the 19th century, the French engineer Darcy experimentally observed the linear relationship for singlephase flow [Darcy, 1856]. For two-phase flow, Darcy's law has been modified to add relative permeabilities which take into account the competition between the phases for available pore space [Bear, 1972].

In general, when two or three phases flow simultaneously through a porous medium, viscous coupling between the phases occurs. It is not well understood whether this coupling effect has a significant contribution to the flow. However, different studies including theoretical [de Gennes, 1983], numerical [Rothman, 1990; Gunstensen and Rothman, 1993] and experimental work [Kalaydjian, 1990; Avraam and Payatakes, 1995] conclude that flows are often better described by a further modification of Darcy's law that includes coupling terms. Avraam et al. [1994] even suggested that both 2D and 3D flow problems are best described by such a modification.

Here, we consider an unidirectional steady-state three-phase flow through a $2 \mathrm{D}$ porous medium. In that case, the three average fluid velocities $u_{k}, k=w$ (water), $o$ (oil) and $g$ (gas), follow the generalized law of Darcy:

$$
\begin{aligned}
& u_{w}=\lambda_{w} X_{w}+\lambda_{w o} X_{o}+\lambda_{w g} X_{g} \\
& u_{o}=\lambda_{o} X_{o}+\lambda_{o w} X_{w}+\lambda_{o g} X_{g} \\
& u_{g}=\lambda_{g} X_{g}+\lambda_{g w} X_{w}+\lambda_{g \sigma} X_{o}
\end{aligned}
$$

where $X_{k}$ is the forcing (a body force minus the pressure gradient of phase $k$ ) in phase $k$. The term $\lambda_{k}$ is the conventional mobility, depending on the pore structure, the fluid parameters (viscosities) and the fluid saturations [Bear, 1972]. The term $\lambda_{\mathrm{kd}}$, the coupling between fluid $\mathrm{k}$ and force $X_{1}$, is assumed to have the same dependency. According to the Onsager-Casimir reciprocal relation, one should find $\lambda_{\mathrm{k}}=\lambda_{\mathrm{lk}}$. However, since this relation is based on microscopic reversibility, this does not have to hold if catastrophic pore-level flow events occur [Avraam and Payatakes, 1995].

\section{Numerical Procedure}

The latice-Boltzmann model is applied to investigate Darcy's law (37). For that purpose, we construct a small porous medium as shown in figures 9 and 10 . The wetting property of the medium is set such that one of the phases (the water phase) completely wets the solid [Rothman, 1990]. In vertical direction, the flow 
domain is bounded by a solid wall. In horizontal direction, a periodic boundary condition is applied. We initially fill the medium randomly such that the three fluid phases have equal saturations. By the horizontal periodicity, these saturations remain fixed. For the forcing $X_{k}$, a horizontal uniform body force is used. This simplifies the investigation of (37) since these forces can be turned on and off for each separate phase. The velocities $u_{k}$ are measured when the system reaches steady-state. For convenience, the fluid viscosities are all taken equal.

Three simulations are needed to determine the nine coefficients in (37). In the first one, $X_{w}$ is assigned a nonzero value, while $X_{0}=X_{0}=0$. In this way, the coefficients $\lambda_{w}, \lambda_{o w}$ and $\lambda_{g w}$ are determined. Similarly, $X_{0}$ and $X_{B}$ are the active forces in respectively the second and third simulation. This provides us values for the other six coefficients (see table 1).

The numerical procedure is applied for a spreading $(S=0)$ and a non-spreading ( $S<0$ ) flow system. $S=0$ is obtained by setting $\gamma_{w_{0}}=\gamma_{o g}=\gamma_{w_{g}} / 2$, while $S<0$ is obtained by setting $\gamma_{w_{0}}=\gamma_{o g}=\gamma_{w g}$. As expected in the case of a spreading system (figure 10), the fluids are more layer-like distributed than in the case of a non-spreading system (figure 9). In figures 9 and 10, the average lluid velocities have become constant in time, apart from small fluctuations. From both figures it is clear that capillary forces are dominated by viscous forces, for the flow is not capable of reaching capillary equilibrium. This is due to the magnitude of the imposed forces $X_{k}$ which is large relative to the mean capillary force.

Table 1: Numerically obtained mobility coefficients (normalized).

\begin{tabular}{c|cc} 
& $S<0$ & $S=0$ \\
\hline$\lambda_{w}$ & $0.35 \pm 0.02$ & $0.37 \pm 0.04$ \\
$\lambda_{\mathrm{o}}$ & $1.01 \pm 0.03$ & $0.78 \pm 0.04$ \\
$\lambda_{\mathrm{s}}$ & $1.05 \pm 0.04$ & $1.59 \pm 0.09$ \\
$\lambda_{\text {wo }}$ & $0.29 \pm 0.03$ & $0.3 \pm 0.02$ \\
$\lambda_{\text {ow }}$ & $0.31 \pm 0.03$ & $0.27 \pm 0.02$ \\
$\lambda_{\text {ws }}$ & $0.3 \pm 0.02$ & $0.23 \pm 0.03$ \\
$\lambda_{\text {gw }}$ & $0.34 \pm 0.01$ & $0.28 \pm 0.02$ \\
$\lambda_{\text {os }}$ & $0.63 \pm 0.02$ & $0.55 \pm 0.04$ \\
$\lambda_{\text {go }}$ & $0.65 \pm 0.04$ & $0.65 \pm 0.04$
\end{tabular}

Table 1 indicates that the Onsager-Casimir reciprocal relation $\lambda_{\mathrm{t}}=\lambda_{1 \mathrm{k}}$ is satisfied in the non-spreading case and, to a lesser degree, in the spreading case. In addition, the coupling coefficients are not significantly affected by the value of $S$, which is also true for the water mobility $\lambda_{w}$. The latter is explained by the fact that water prefers to wet the solid, not being sensitive to the presence of the other phases.

Since all the interfacial tensions are equal in the nonspreading case, there is no reason for the oil mobility $\lambda_{\text {o }}$ to differ from the gas mobility $\lambda_{k}$ (recall that the three fluid saturations are equal). This is numerically confirmed and shown in table 1 . In the spreading case, however, it is energetically unfavourable to maintain gas-water interfaces. This can be seen in figure 10 in which the oil phase separates gas and water almost everywhere. In other words, gas prefers to flow in the center of the pore space, while oil prefers to flow closer to the solid. This explains the numerical findings that $\lambda_{\mathrm{s}}$ is larger for $S=0$ than for $S<0$, and that $\lambda_{0}$ is smaller for $S=0$ than for $S<0$.

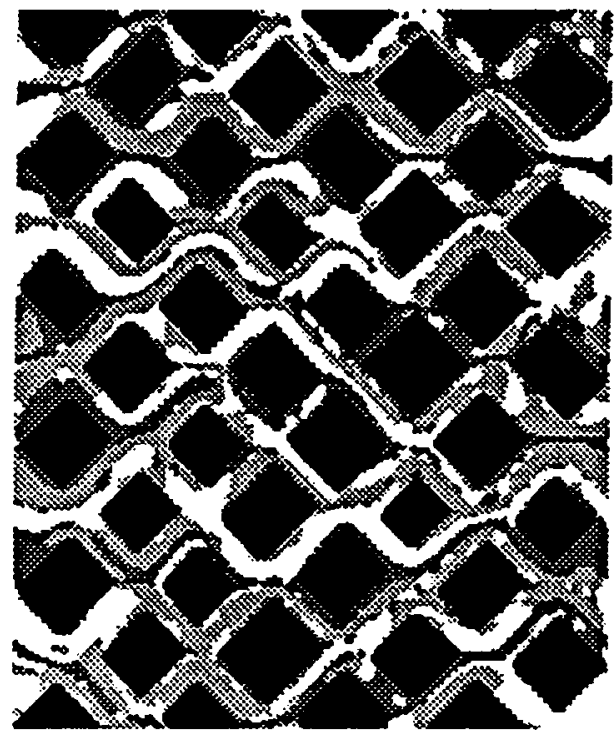

Figure 9: Flow of oil (white), gas (light grey) and water (dark grey) in a porous medium (black grains) for $S<0$ and $X_{w}=X_{o}=X_{s}=X \neq 0$ (in horizontal direction).

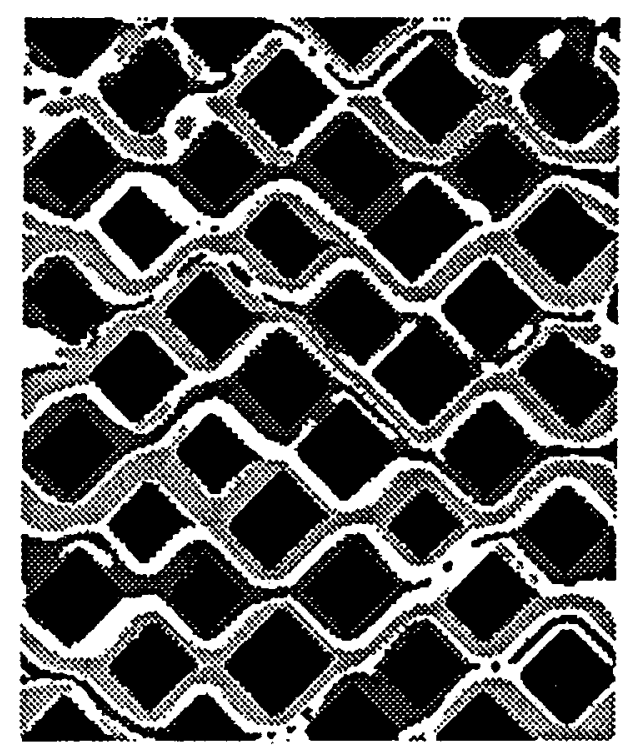

Figure 10: Flow of oil (white), gas (light grey) and water (dark grey) in a porous medium (black grains) for $S=0$ and $\mathrm{X}_{\mathrm{w}}=\mathrm{X}_{\mathrm{o}}=\mathrm{X}_{\mathrm{B}}=\mathrm{X} \neq 0$ (in horizontal direction).

A fourth simulation is performed, in which $X_{w}=X_{0}=X_{2}$ $=X \neq 0$, to test whether the flow can be predicted by the generalized law of Darcy (37) with the coefficients from table 1. This test consists of comparing the predicted fluid velocities to the corresponding measured velocities. Table $2(S<0)$ and table $3(S=0)$ show that the generalized law 
of Darcy gives an adequate description for the considered flow.

We realize that the relative importance of the coupling terms may diminish if other saturation values are chosen and/or if the extension to 3D flows is made.

Table 2: Predicted versus measured velocities (normalized) for $S<0$.

\begin{tabular}{c|cc} 
& predicted & measured \\
\hline$u_{w}$ & $0.94 \pm 0.07$ & $1.05 \pm 0.07$ \\
$u_{o}$ & $1.95 \pm 0.08$ & $2.12 \pm 0.08$ \\
$u_{\mathrm{g}}$ & $2.04 \pm 0.09$ & $2.16 \pm 0.14$
\end{tabular}

Table 3: Predicted versus measured velocities (normalized) for $S=0$.

\begin{tabular}{c|cc} 
& predicted & measured \\
\hline $\mathrm{u}_{w}$ & $0.9 \pm 0.09$ & $1.05 \pm 0.01$ \\
$\mathrm{u}_{\mathrm{o}}$ & $1.6 \pm 0.1$ & $1.72 \pm 0.06$ \\
$\mathrm{u}_{\mathrm{g}}$ & $2.52 \pm 0.15$ & $2.39 \pm 0.04$
\end{tabular}

\section{CONCLUSIONS}

The latice-Boltzmann model is capable of simulating three-phase flow at the pore level. Two situations can occur at a three-phase intersection. For nonspreading conditions, stable contact angles exist (see figure 1), while for spreading conditions, the oil phase spreads between the gas and water phase (see figure 3 ). As a verification, the spreading of an oil droplet onto the gas-water interface has been simulated. It turned out that the radius of the droplet follows a power law (equations 19 and 35) for capillary driven spreading. The simulation results agree with theoretical and experimental findings from the literature.

We also simulated steady-state three-phase flow (2D) through a small waterwet porous medium, in a regime where viscous forces dominate all other forces. It was found that this kind of flow is best described by the generalized law of Darcy (equation 37) which accounts for viscous coupling. In the spreading case, it is energetically unfavourable to maintain gas-water interfaces. This has been confirmed by the simulations, where it is observed that oil separates gas and water (see figure 10). In the nonspreading case, a fluid phase is not systematically separated from any other fluid phase because of the occurrence of contact angles (see figure 9). Further, it was shown that the spreading coefficient does not significantly affect the coupling coefficients and the water mobility $\lambda_{w}$ (see table 1). The simulations confirm the OnsagerCasimir reciprocal relation for both the spreading and the non-spreading case.

\section{ACKNOWLEDGMENTS}

This research has been funded in part by the European Commission in the context of the Brite Euram research programme.

\section{REEERENCES}

1. Avraam, D.G., Kolonis, G.B., Roumeliotis, T.C., Constantinides, G.N. \& Payatakes, A.C., 1994, Steady-state two-phase flow through planar and nonplanar model porous media, Transport in Porous Media 16, 75-101.

2. Avraam, D.G. \& Payatakes, A.C., 1995, Generalized relative permeability coefficients during steady-state two-phase flow in porous media, and correlations with the flow mechanisms, Transport in Porous Media 20, 135-168.

3. Baker, L.E., 1988, Three-phase relative permeability correlations, SPE 17369, 539-554.

4. Bear, J., 1972, Dynamics of fluids in porous media, Dover, New York.

5. Borgas, M.S. \& Grotberg, J.B., 1988, Monolayer flow on a thin film, J. Fluid Mech. 193, 151-170.

6. Chatzis, I., Kantzas, A. \& Dullien, F.A.L., 1988, On the investigation of gravity assisted inert gas injection using micromodels, long Berea cores, and computer assisted tomography, SPE 18284.

7. Chen, H., Chen, S. \& Matthaeus, W.H., 1992, Recovery of the Navier-Stokes equations using a lattice-gas Boltzmann method, Phys. Rev. A 45 (8), R5339.

8. Darcy, $\mathrm{H}$., 1856, Les fontaines publiques de la ville de Dijon, Victor Dalmot, Paris.

9. de Gennes, P., 1983, Theory of slow biphasic flows in porous media, Phys. Chem. Hydrodyn. 4, 175185.

10. Fraaije, J.G.E.M. \& Cazabat, A.M., 1989, Dynamics of spreading on a liquid substrate, J. Colloid Interface Sci. 133 (2), 452-460.

11. Frisch, U., d'Humières, D., Hasslacher, B., Lallemand, P., Pomeau, Y. \& Rivet, J.P., 1987, Lattice gas hydrodynamics in two and three dimensions, Complex Syst. 1, 649.

12. Gaver III, D.P. \& Grotberg, J.B., 1992, Droplet spreading on a thin viscous film, J. Fluid Mech. 235, 399-414.

13. Ginzbourg, I., 1994, Les problèmes de conditions aux limites dans les méthodes de gaz sur réseaux à plusieurs phases, Thesis.

14. Grunau, D.W., 1993, Lattice methods for modeling hydrodynamics, Thesis.

15. Gunstensen, A.K. \& Rothman, D.H., 1991, A lattice-gas model for three immiscible fluids, Physica D 47, 47-52.

16. Gunstensen, A.K. \& Rothman, D.H., 1993, LatticeBoltzmann studies of immiscible two-phase flow through porous media, Journal of Geophysical Research 98 (B4), 6431-6441.

17. Gunstensen, A.K., Rothman, D.H., Zaleski, S. \& Zanetti, G., 1991, Lattice Boltzmann model of immiscible fluids, Physical Review A 43 (8), 43204327. 
18. Joanny, J.F., 1987, Wetting of a liquid substrate, Physico Chemical Hydrodynamics 9, 183-196.

19. Kalaydjian, F.J-M., 1990, Origin and quantification of coupling between relative permeabilities for twophase flows in porous media, Transport in Porous Media 5, 215-229.

20. Kalaydjian, F.J-M., Moulu, J-C. \& Vizika, O., 1993, Three-phase flow in water-wet porous media: Determination of gas/oil relative permeabilities under various spreading conditions, SPE 26671, 689-700.

21. Koch, D.M. \& Koch, D.L., 1995, Numerical and theoretical solutions for a drop spreading below a free fluid surface, J. Fluid Mech. 287, 251-278.

22. Oak, MJ., 1990, Three-phase relative permeability of waterwet Berea, SPE/DOE 20183, 109-120.

23. Øren, P.E. \& Pinczewski, W.V., 1995, Fluid distribution and pore-scale displacement mechanisms in drainage dominated three-phase flow, Transport in Porous Media 20 (1 \& 2), 105-133.

24. Rothman, D.H., 1990, Macroscopic laws for immiscible two-phase flow in porous media: Results from numerical experiments, Journal of Geophysical Research 95 (B6), 8663-8674.

25. Rowlinson, J.S. \& Widom, B., 1982, Molecular theory of capillarity.

26. Stone, H.L., 1973, Estimation of three-phase relative permeability and residual oil data, Journal of Canadian Petroleum Technology 12, 53-61. 